\title{
P 031 TITRATION OF ABSTRAL IN THE HOSPICE SETTING: HOW DOES ABSTRAL DOSE RELATE TO BACKGROUND OPIOID REQUIREMENT?
}

Adam Hurlow, ${ }^{1}$ Clare Rayment, ${ }^{2}$ Jason Ward ${ }^{3} .{ }^{1}$ Palliative Care Team, Leeds Teaching Hospitals NHS Trust, Leeds, United Kingdom; ${ }^{2}$ Marie Curie Hospice, Leeds, United Kingdom; ${ }^{3}$ St Gemma's Hospice, Leeds, United Kingdom

10.1136/bmjspcare-2014-000654.72

Background Sublingual fentanyl citrate orally disintegrating table (Abstral, ODT) is a rapid onset opioid developed for breakthrough cancer pain. Whilst ODT may be more efficacious than oral morphine for breakthrough pain the titration process of starting on the lowest dose may limit its use.

Aims The aims of this study were to identify how many patients were titrated to an effective ODT maintenance dose (stable for at least a week), to compare the maintenance dose to the background opioid dose at the time ODT was started and to identify reasons for unsuccessful titration.

Methods We conducted a retrospective notes review of the first 30 hospice inpatients to be commenced on ODT in a 32 bed UK hospice. All opioid doses are expressed as the oral morphine equivalent calculated using hospice guidelines.

Results All patients had cancer and their median age was 64.5 (48-88) years. Seventeen patients achieved a maintenance dose and 12 continued ODT until death/data collection. In eight patients titration failed because the patient started to die. The maintenance ODT dose was $400 \mathrm{mcg}$ or less in ten patients and $600-800 \mathrm{mcg}$ in seven patients. The median background opioid doses were $205 \mathrm{mg}$ and $500 \mathrm{mg}$ respectively. The median titration period was $4(0-20)$ days with only two patients taking more than a week. In three patients toxicity was the reason for stopping ODT.

Conclusions Abstral ODTwas successfully titrated and effective in approximately half of all patients. Patients who required ODT doses of $600 \mathrm{mcg}$ or more had a higher median background opioid dose suggesting a relationship between the two. Although titration to a maintenance dose took less than a week in almost all patients, onset of dying was the most common reason for titration failure. This suggests the titration process may be problematic for patients with short prognoses necessitating robust patient selection. 